\title{
Advanced chondrosarcoma of the pelvis: A rare case of urinary obstruction
}

\author{
Valerio Olivieri ${ }^{1}$, Valentina Fortunati ${ }^{1}$, Scipio Annoscia ${ }^{1}$, Massimo Massarelli ${ }^{1}$, Luca Bellei ${ }^{1}$, \\ Massimo Ollino ${ }^{1}$, Emy Manzi ${ }^{2}$, Angela Maurizi ${ }^{3}$, Francesco De Luca ${ }^{3}$ \\ ${ }^{1}$ Urology Department, Ivrea Hospital - ASL TO4, Ivrea, Turin, Italy; \\ 2 Department of General Surgery, Santa Scolastica Hospital Manzi, Cassino (FR), Italy; \\ ${ }^{3}$ Department of Gynaecological and Urological Sciences, Sapienza University of Rome, Rome, Italy.
}

\begin{abstract}
Summary Chondrosarcoma is the second most common malignant tumor of the bone with an incidence of 1 in 200.000 per year. Axial skeleton is frequently involved showing poorer oncological outcomes than appendicular one: human pelvis is a site predilection (1).

It is rarely associated to urinary obstruction but according to its localization, it can be frequently linked to compression of pelvic organs as bladder, prostate or bowel. We describe the case of a 52 years old caucasian male with history of advanced pelvic chondrosarcoma and severe hydronephrosis due to total bladder dislocation.
\end{abstract}

KEY WORDS: Urology; Urinary obstruction; Hydronephrosis; Bladder dislocation; Pelvic chondrosarcoma.

Submitted 13 August 2018; Accepted 19 August 2018

\section{Case report}

A 52 years old feeble-minded male presented to our facility for bilateral flank pain poorly responsive to nonsteroidal anti-inflammatory therapy. The patient was previously diagnosed with pelvic chondrosarcoma but due to his general conditions, the advanced state of the tumor and its localization, no further therapies were offered to him just suggesting best supportive care. He has suffered for several months but pain intensified during last weeks: the pain was described as severe, originating in the flank bilaterally, irradiating anteriorly and associated to nausea and vomiting. Anyway he did not give history of urolithiasis, haematuria or previous flank pain episodes: no bowel diseases were mentioned. He solely referred a contraction of the diuresis which occurred during the last four weeks without any lower urinary tract symptoms. On physical examination he was alert and afebrile. The abdomen was soft with Giordano's test fully positive on bilateral flank: no signs of acute urinary retention was found neither evoked pain in hypogastric region. Blood examination revealed severe renal failure with creatinine on the high values; anyway white blood cells and haemoglobin were both normal. Abdominal ultrasound showed a voluminous bilateral renal pelvis with short residual parenchyma, highly suggestive for chronic distal obstruction. Moreover bladder ultrasound was inconclusive being unable to visualize the pelvic organ due to skeleton artifacts due to his bone pathology.

Due to the discovery of bilateral hydronephrosis, the inability of bladder visualizing and the presence of a pelvic bone tumor, the patient underwent to no-contrast computerized tomography (CT) in order to exclude ureteric compression by the tumor: no iodinated contrast material was given due to renal failure.

The exam confirmed a voluminous bilateral hydronephrosis and hydroureter highly suggestive for chronic distal obstruction (Figure 1). No signs of urinary stones in both ureters were described. The scan also revealed a total bladder dislocation due to the pelvic chondrosarcoma (Figure 2) originating from left ischiopubic ramus and occupying almost the entire pelvis (Figure 3).

As compared with a previous computerized tomography, it resulted highly increased in size and the bladder, which was previously orthotopic, was now entirely located and compressed in the upper part of the right pelvis. No visceral metastases were detected on tomography. Patient urgently underwent bilateral nephrostomy tubes placing in order to drain hydronephrosis, improve renal failure and alleviate the flank pain.

\section{Figure 1.}

Bilateral hydronephrosis due to urinary obstruction.

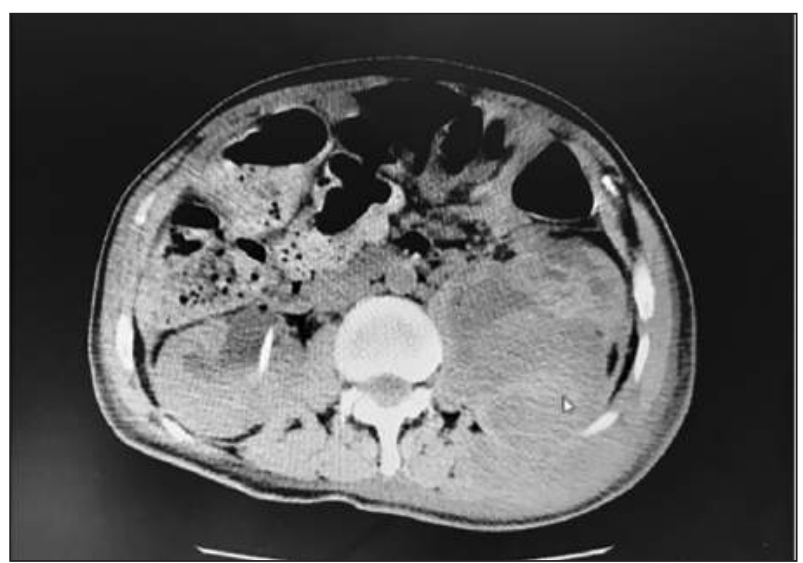


Figure 2.

Total bladder dislocation due to pelvic chondrosarcoma.

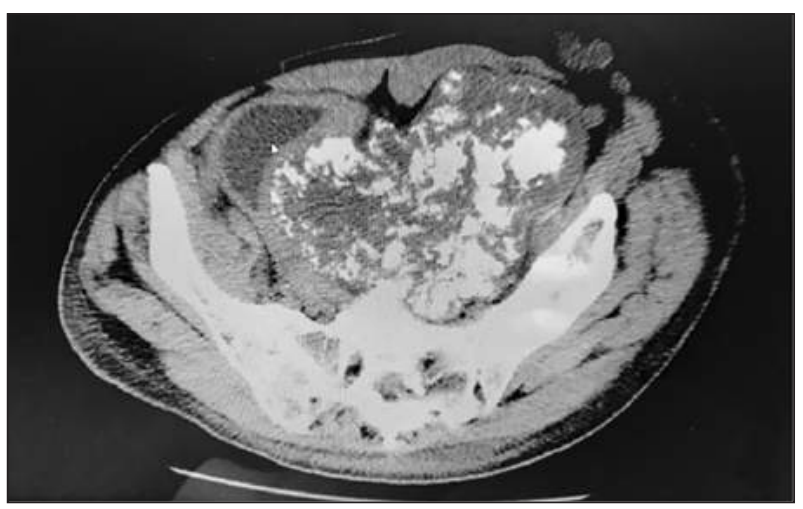

Figure 3.

Pelvic chondrosarcoma originating from left ischiopubic ramus.

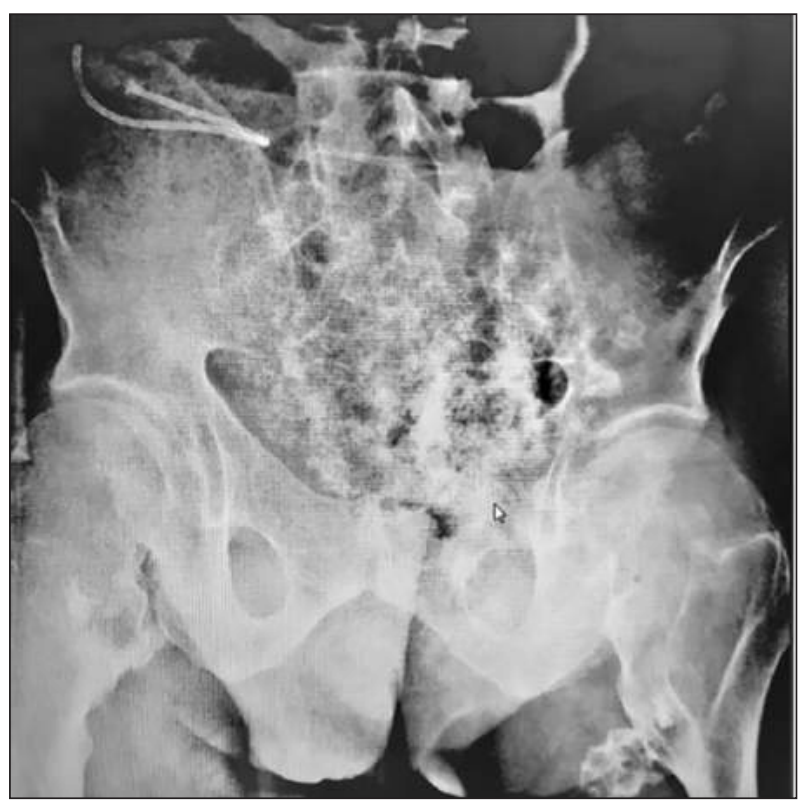

\section{Discussion}

Chondrosarcoma is a malignant tumor of the bone originating from axial or appendicular skeleton. Femur and proximal humerus are frequently involved as site of predilection: pelvis localization is also much common and this represent a major risk factor for pelvic organs compression. Bladder, bowel or major vessels may be easily involved but to our knowledge this is the first case in literature associated with severe bilateral hydronephrosis and total bladder dislocation. Many authors have shown how a wide tumor resection is essential in the therapy of primary bone neoplasms in order to ensure long-term survival particularly in chondrosarcoma but tumor localization and pelvic organs involving may result as a challenging surgical case (2). Anyway proper selection of patient, preoperative planning and reconstruction provides good functional outcome also in patients affected by pelvic chondrosarcoma following internal hemipelvectomy (3). In our case, due to general conditions and tumor extension, the patient was considered as unfit for any major surgical approach simply receiving palliative nephrostomy.

\section{Conclusions}

Pelvic chondrosarcoma have been rarely shown to cause total bladder dislocation and bilateral hydronephrosis but according to the risk of obstructive renal failure, the work-up of patient affected by this tumor should include a regular monitoring of renal function and mass growth.

\section{REFERENCES}

1. Mavrogenis AF, Angelini A, Drago G, et al. Survival analysis of patients with chondrosarcomas of the pelvis. J Surg Oncol. 2013; 108:19-27.

2. Guder WK, Hardes J, Gosheger M, et al. Osteosarcoma and chondrosarcoma of the pelvis and lower extremities. Chirurg. 2015; 86:993-1003.

3. Salunke AA, Shah J, Warikoo V, et al. Surgical management of pelvic bone sarcoma with internal hemipelvectomy: oncologic and functional outcomes. J Clin Orthop Trauma. 2017; 8:249-253.

\author{
Correspondence \\ Valerio Olivieri, MD \\ valerio.olivieri@uniromal.it \\ Valentina Fortunati, MD \\ valentina.fortunati@email.it \\ Scipio Annoscia, MD \\ sannoscia@aslto4.piemonte.it \\ Massarelli Massimo, MD \\ mmassarelli@gmail.com \\ Luca Bellei, MD \\ lbellei@aslto4.piemonte.it \\ Massimo Ollino, MD \\ mollino@aslto4.piemonte.it \\ Sandro Guglielmetti, MD \\ gugls76@yahoo.it \\ Urology Dpt, Ivrea Hospital - ASL TO4, \\ Piazza Credenza 2, 10015 Ivrea, Turin, Italy
}

Emy Manzi, MD

emymanzi@gmail.com

Department of General Surgery, Santa Scolastica Hospital

Manzi, Via San Pasquale, 03043 Cassino (FR), Italy

Angela Maurizi, MD

angmau81@hotmail.com

Francesco De Luca, MD (Corresponding Author)

francescodeluca.md@gmail.com

Department of Gynaecological and Urological Sciences, Sapienza University Viale dell'Università, 31/33, 00161 Roma, Italy 